\title{
A New Improved Parsimonious Multivariate Markov Chain Model
}

\author{
Chao Wang and Ting-Zhu Huang \\ School of Mathematical Sciences, University of Electronic Science and Technology of China, Chengdu, Sichuan 611731, China
}

Correspondence should be addressed to Chao Wang; wangchao1321654@163.com

Received 12 November 2012; Revised 17 December 2012; Accepted 2 January 2013

Academic Editor: Marco H. Terra

Copyright (C) 2013 C. Wang and T.-Z. Huang. This is an open access article distributed under the Creative Commons Attribution License, which permits unrestricted use, distribution, and reproduction in any medium, provided the original work is properly cited.

We present a new improved parsimonious multivariate Markov chain model. Moreover, we find a new convergence condition with a new variability to improve the prediction accuracy and minimize the scale of the convergence condition. Numerical experiments illustrate that the new improved parsimonious multivariate Markov chain model with the new convergence condition of the new variability performs better than the improved parsimonious multivariate Markov chain model in prediction.

\section{Introduction}

The probability distribution of Markov chains plays an important role in a wide range of applications such as telecommunication systems, manufacturing systems, and inventory systems, see, for instance, [1] and the references therein. In recent years, the prediction of multivariate Markov chain models [2] has become more and more useful in many realworld applications: sales demand predictions $[2,3]$, DNA sequencing [4], and credit and financial data modeling [5]. The major merit of the multivariate Markov chain model is to detect the relations among the sequences and to predict more precisely.

Different models for multiple categorical data sequences are introduced in the following part. A multivariate Markov chain model has been presented in [2]. Ching et al. constructed a new matrix by means of the transition probability matrices among different sequences. To improve prediction accuracy, Ching et al. incorporated positive and negative parts in an improved parsimonious multivariate Markov chain model [5]. Miao and Hambly presented recursive formulas for the default probability distribution which is feasible for computation in this simple version [6]. A more advanced model, namely, higher-order multivariate Markov chain model has been exhibited in [7]. To reduce the number of parameters of the model, a parsimonious higher-order multivariate Markov chain model has been proposed in [8], where the number of parameters is $\mathcal{O}\left((n+s) s m^{2}\right)$. Certainly, there are many other papers contributing to the multivariate Markov chain models, for example, $[1,9,10]$.

With the development of science technologies with their applications, the number of data in sequences become larger, and the results need to be more precise. It is inevitable that a large categorical data sequence group will cause high computational costs, especially using the convergence condition as in [5]. In this paper, a new improved parsimonious multivariate Markov chain model and a new convergence condition with a new variability are presented to enhance the precision of the prediction and save the computational costs.

The rest of the paper is organized as follows. In Section 2, we briefly review several multivariate Markov chain models. In Section 3, a new improved parsimonious multivariate Markov chain model and a new convergence condition with a new variability are presented. Section 4 gives the estimation methods for the parameters of the new improved parsimonious multivariate Markov chain model with different convergence conditions. Numerical experiments with three examples are presented to demonstrate the effectiveness of our proposed model with the new convergence condition of the new variability in Section 5. Finally, concluding remarks are given in Section 6. 


\section{Review of the Multivariate Markov Chain Models}

In this section, we briefly introduce several multivariate Markov chain models, for example, the Markov chain model [3], the multivariate Markov chain model [2], and the improved parsimonious multivariate Markov chain model [5].

2.1. The Markov Chain Model. First, we introduce some definitions of the Markov chain [2,11]. Let the state set of the categorical data sequences be $\mathscr{M}=\{1,2, \ldots, m\}$. The discrete-time Markov chain with $m$ states satisfies the following relations:

$$
\begin{gathered}
\operatorname{Prob}\left(x_{t+1}=\theta_{t+1} \mid x_{0}=\theta_{0}, x_{1}=\theta_{1}, \ldots, x_{t}=\theta_{t}\right) \\
=\operatorname{Prob}\left(x_{t+1}=\theta_{t+1} \mid x_{t}=\theta_{t}\right),
\end{gathered}
$$

where $\theta_{t} \in \mathscr{M}, t \in\{0,1,2, \ldots\}$. The conditional probability $\operatorname{Prob}\left(x_{t+1}=\theta_{t+1} \mid x_{t}=\theta_{t}\right)$ is called the one-step transition probability of the Markov chain. The transition probability is

$$
P^{(i, j)}=\operatorname{Prob}\left(x_{t+1}=i \mid x_{t}=j\right), \quad \forall i, j \in \mathscr{M},
$$

and the transition probability matrix is $P^{(i, j)}$. The Markov chain model can be represented as follows:

$$
X_{t+1}=P X_{t}
$$

where

$$
\begin{aligned}
& P=\left[P^{(i, j)}\right], \quad 0 \leq P^{(i, j)} \leq 1, \forall i, j \in \mathscr{M}, \\
& \sum_{i=1}^{m} P^{(i, j)}=1, \quad \forall j \in \mathscr{M},
\end{aligned}
$$

$X_{0}$ is the initial probability distribution, and $X_{t}=$ $\left(x_{t}^{1}, x_{t}^{2}, \ldots, x_{t}^{m}\right)^{\mathrm{T}}$ is the state probability distribution at time $t$.

2.2. The Multivariate Markov Chain Model. Suppose the number of categorical data sequences $s>1$. The multivariate Markov chain model [2] is represented as follows:

$$
x_{t+1}^{(j)}=\sum_{k=1}^{s} \lambda_{j, k} P^{(j, k)} x_{t}^{(k)}, \quad \forall j=1,2, \ldots, s, t=0,1, \ldots,
$$

where

$$
\begin{aligned}
& \lambda_{j, k} \geq 0, \quad \forall j, k=1,2, \ldots, s, \\
& \sum_{k=1}^{s} \lambda_{j, k}=1, \quad \forall j=1,2, \ldots, s .
\end{aligned}
$$

Here, $x_{0}^{(j)}$ is the initial probability distribution of the $j$ th sequence, $x_{t}^{(k)}$ is the state probability distribution of the $k$ th sequence at time $t$, and $x_{t+1}^{(j)}$ is the state probability distribution of the $j$ th sequence at time $t+1$. Here, $P^{(j, k)}$ is the one-step transition probability from the state in the $k$ th sequence at time $t$ to the state in the jth sequence at time $t+1$. In matrix form, we have

$$
\begin{aligned}
X_{t+1}=\left(\begin{array}{c}
x_{t+1}^{(1)} \\
x_{t+1}^{(2)} \\
\vdots \\
x_{t+1}^{(s)}
\end{array}\right)= & \left(\begin{array}{cccc}
\lambda_{1,1} P^{(1,1)} & \lambda_{1,2} P^{(1,2)} & \cdots & \lambda_{1, s} P^{(1, s)} \\
\lambda_{2,1} P^{(2,1)} & \lambda_{2,2} P^{(2,2)} & \cdots & \lambda_{2, s} P^{(2, s)} \\
\vdots & \vdots & \vdots & \vdots \\
\lambda_{s, 1} P^{(s, 1)} & \lambda_{s, 2} P^{(s, 2)} & \cdots & \lambda_{s, s} P^{(s, s)}
\end{array}\right) \\
& \times\left(\begin{array}{c}
x_{t}^{(1)} \\
x_{t}^{(2)} \\
\vdots \\
x_{t}^{(s)}
\end{array}\right) .
\end{aligned}
$$

Here, $P^{(j, k)}$ and $\lambda_{j, k}$ can be obtained from the $s$ categorical data sequences and the corresponding linear programming, for details, refer to [2].

2.3. The Improved Parsimonious Multivariate Markov Chain Model. With the same notations as introduced in Section 2.2, we introduce the improved parsimonious multivariate Markov chain model originating from multivariate Markov chain model. Consider

$$
Z_{t+1}=\frac{1}{m-1}\left(\mathbf{e}-X_{t}\right),
$$

where the factor $1 /(m-1)$ is a constant for normalization. Here, $\mathbf{e}$ is the vector of all ones and $m \geq 2$. In matrix form, the improved parsimonious multivariate Markov chain model can be represented as

$$
\left(\begin{array}{c}
x_{t+1}^{(1)} \\
x_{t+1}^{(2)} \\
\vdots \\
x_{t+1}^{(s)}
\end{array}\right)=\Lambda^{+}\left(\begin{array}{c}
x_{t}^{(1)} \\
x_{t}^{(2)} \\
\vdots \\
x_{t}^{(s)}
\end{array}\right)+\frac{1}{m-1} \Lambda^{-}\left(\begin{array}{c}
\mathbf{e}-x_{t}^{(1)} \\
\mathbf{e}-x_{t}^{(2)} \\
\vdots \\
\mathbf{e}-x_{t}^{(s)}
\end{array}\right)
$$

where

$$
\begin{gathered}
\Lambda^{+}=\left(\begin{array}{cccc}
\lambda_{1,1} P^{(1,1)} & \lambda_{1,2} P^{(1,2)} & \cdots & \lambda_{1, s} P^{(1, s)} \\
\lambda_{2,1} P^{(2,1)} & \lambda_{2,2} P^{(2,2)} & \cdots & \lambda_{2, s} P^{(2, s)} \\
\vdots & \vdots & \vdots & \vdots \\
\lambda_{s, 1} P^{(s, 1)} & \lambda_{s, 2} P^{(s, 2)} & \cdots & \lambda_{s, s} P^{(s, s)}
\end{array}\right) \\
\Lambda^{-}=\left(\begin{array}{cccc}
\lambda_{1,-1} P^{(1,1)} & \lambda_{1,-2} P^{(1,2)} & \cdots & \lambda_{1,-s} P^{(1, s)} \\
\lambda_{2,-1} P^{(2,1)} & \lambda_{2,-2} P^{(2,2)} & \cdots & \lambda_{2,-s} P^{(2, s)} \\
\vdots & \vdots & \vdots & \vdots \\
\lambda_{s,-1} P^{(s, 1)} & \lambda_{s,-2} P^{(s, 2)} & \cdots & \lambda_{s,-s} P^{(s, s)}
\end{array}\right), \\
\sum_{j=-s}^{s} \lambda_{j, k}=1, \quad \lambda_{j, k} \geq 0, \text { for } \forall 1 \leq j \leq s, 1 \leq|k| \leq s .
\end{gathered}
$$


$\Lambda^{+} X_{t}$ and $\Lambda^{-} Z_{t}$ are the positive and negative parts of the transition probability matrices in (9). The above model has two directions to approach the steady solution $X$.

Let $A=\left[a_{i, j}\right] \in \mathbb{R}^{m \times m}, B=\left[b_{i, j}\right] \in \mathbb{R}^{m \times m}$. If $\left|a_{i, j}\right| \geq b_{i, j}$ for for all $i, j=\{1,2, \ldots, m\}$ and there exist $i, j \in\{1,2, \ldots, m\}$ satisfying $\left|a_{i, j}\right|>b_{i, j}$, then we note that $|A|>B$.

Lemma 1 (see [12]). Let $A \in \mathbb{R}^{m \times m}$ be a nonnegative and irreducible matrix, $B \in \mathbb{C}^{m \times m}$ a complex matrix, and $\lambda$ an eigenvalue of $B$. If $|A|>B$, then $\rho(A)>|\lambda|$.

\section{A New Improved Parsimonious Multivariate Markov Chain Model}

In this section, we propose a new improved parsimonious multivariate Markov chain model and a new convergence condition with a new variability.

In the new model, the state probability distribution of the $j$ th sequence at time $t+1$ depends on the state probability distribution of all the sequences at time $t$. Let the number of categorical data sequences be $s>1, m$ the number of states in every sequences, $\beta<1 / m$, then the new improved parsimonious multivariate Markov chain model can be represented as follows:

$$
X_{t+1}^{(j)}=\sum_{k=1}^{s} \lambda_{j, k} P^{(j, k)} X_{t}^{(k)}+\frac{1}{m \beta-1} \sum_{k=1}^{s} \lambda_{j,-k} P^{(j, k)}\left(\beta \mathbf{e}-X_{t}^{(k)}\right),
$$

where $X_{0}^{(k)}$ is the initial probability distributions of the $k$ th sequence and

$$
\sum_{k=-s}^{s} \lambda_{j, k}=1, \quad \lambda_{j, k} \geq 0, \text { for } \forall 1 \leq j \leq s, 1 \leq|k| \leq s .
$$

Here, $X_{t}^{(k)}$ is the state probability distribution of the $k$ th sequence at time $t, P^{(j, k)}$ is the one-step transition probability matrix from the state in the $k$ th sequence at time $t$ to the state in the $j$ th sequence at time $t+1$. Here, $X_{t+1}^{(j)}$ is the state probability distribution of the $j$ th sequence at time $t+1$. Let

$$
X_{t+1}=\left(\left(X_{t+1}^{(1)}\right)^{\mathrm{T}},\left(X_{t+1}^{(2)}\right)^{\mathrm{T}}, \ldots\left(X_{t+1}^{(s)}\right)^{\mathrm{T}}\right)^{\mathrm{T}} \in \mathbb{R}^{m s \times 1},
$$

then the new improved parsimonious multivariate Markov chain model in matrix form is

$$
X_{t+1}=\Lambda^{+} X_{t}+\frac{1}{m \beta-1} \Lambda^{-}\left(\beta \mathbf{e}-X_{t}\right),
$$

which also can be represented as

$$
\left(\begin{array}{c}
X_{t+1}^{(1)} \\
X_{t+1}^{(2)} \\
\vdots \\
X_{t+1}^{(s)}
\end{array}\right)=\Lambda^{+}\left(\begin{array}{c}
X_{t}^{(1)} \\
X_{t}^{(2)} \\
\vdots \\
X_{t}^{(s)}
\end{array}\right)+\frac{1}{m \beta-1} \Lambda^{-}\left(\begin{array}{c}
\beta \mathbf{e}-X_{t}^{(1)} \\
\beta \mathbf{e}-X_{t}^{(2)} \\
\vdots \\
\beta \mathbf{e}-X_{t}^{(s)}
\end{array}\right),
$$

where

$$
\begin{gathered}
\Lambda^{+}=\left(\begin{array}{cccc}
\lambda_{1,1} P^{(1,1)} & \lambda_{1,2} P^{(1,2)} & \cdots & \lambda_{1, s} P^{(1, s)} \\
\lambda_{2,1} P^{(2,1)} & \lambda_{2,2} P^{(2,2)} & \cdots & \lambda_{2, s} P^{(2, s)} \\
\vdots & \vdots & \vdots & \vdots \\
\lambda_{s, 1} P^{(s, 1)} & \lambda_{s, 2} P^{(s, 2)} & \cdots & \lambda_{s, s} P^{(s, s)}
\end{array}\right), \\
\Lambda^{-}=\left(\begin{array}{cccc}
\lambda_{1,-1} P^{(1,1)} & \lambda_{1,-2} P^{(1,2)} & \cdots & \lambda_{1,-s} P^{(1, s)} \\
\lambda_{2,-1} P^{(2,1)} & \lambda_{2,-2} P^{(2,2)} & \cdots & \lambda_{2,-s} P^{(2, s)} \\
\vdots & \vdots & \vdots & \vdots \\
\lambda_{s,-1} P^{(s, 1)} & \lambda_{s,-2} P^{(s, 2)} & \cdots & \lambda_{s,-s} P^{(s, s)}
\end{array}\right), \\
\sum_{k=-s}^{s} \lambda_{j, k}=1, \quad \lambda_{j, k} \geq 0, \text { for } \forall 1 \leq j \leq s, 1 \leq|k| \leq s .
\end{gathered}
$$

$\Lambda^{+} X_{t}$ and $(1 /(m \beta-1)) \Lambda^{-}\left(\mathbf{e} \beta-X_{t}\right)$ are, respectively, the positive and negative parts of the transition probability matrices in (14) where $\left\|X_{t}\right\|_{1}=\left\|(1 /(m \beta-1))\left(\mathbf{e} \beta-X_{t}\right)\right\|_{1}=1$. Each column sum of $P^{(j, k)}$ is equal to one.

From (14), after $t$ times iterations, it has

$$
\begin{aligned}
X_{t+1}= & \Lambda^{+} X_{t}+\frac{1}{m \beta-1} \Lambda^{-}\left(\beta \mathbf{e}-X_{t}\right) \\
= & \left(\Lambda^{+}-\frac{1}{m \beta-1} \Lambda^{-}\right) X_{t}+\frac{\beta}{m \beta-1} \Lambda^{-} \mathbf{e} \\
= & \left(\Lambda^{+}-\frac{1}{m \beta-1} \Lambda^{-}\right)\left(\left(\Lambda^{+}-\frac{1}{m \beta-1} \Lambda^{-}\right) X_{t-1}\right. \\
& \left.\quad+\frac{\beta}{m \beta-1} \Lambda^{-} \mathbf{e}\right)+\frac{\beta}{m \beta-1} \Lambda^{-} \mathbf{e} \\
= & \left(\Lambda^{+}-\frac{1}{m \beta-1} \Lambda^{-}\right)^{2} X_{t-1} \\
& +\left(\Lambda^{+}-\frac{1}{m \beta-1} \Lambda^{-}\right) \frac{\beta}{m \beta-1} \Lambda^{-} \mathbf{e}+\frac{\beta}{m \beta-1} \Lambda^{-} \mathbf{e} \\
= & \left(\Lambda^{+}-\frac{1}{m \beta-1} \Lambda^{-}\right)^{t+1} X_{0} \\
& +\sum_{k=0}^{t}\left(\Lambda^{+}-\frac{1}{m \beta-1} \Lambda^{-}\right)^{k} \frac{\beta}{m \beta-1} \Lambda^{-} \mathbf{e} .
\end{aligned}
$$

If $\rho\left(\Lambda^{+}-(1 /(m \beta-1)) \Lambda^{-}\right)<1$, the iteration of the new improved parsimonious multivariate Markov chain model is convergent. For finding a more simple and efficient convergence condition from the point of view of properties of special matrices, we get the following theorem.

Theorem 2. In the new improved parsimonious multivariate Markov chain model, if $\Lambda^{+}>(\gamma /(m \beta-1)) \Lambda^{-}$where $\gamma \geq 1$ and $\beta<1 / m$, then the iteration of the new improved parsimonious multivariate Markov chain model is convergent. 
Proof. Because $\Lambda^{+}>(\gamma /(m \beta-1)) \Lambda^{-}$, we obtain

$$
\Lambda^{+}-\frac{\gamma}{m \beta-1} \Lambda^{-}>0
$$

For $\Lambda^{+}, \Lambda^{-}>0$, it has

$$
-\Lambda^{+}<0<\Lambda^{+}-\frac{\gamma}{m \beta-1} \Lambda^{-}<\Lambda^{+} .
$$

Then, we obtain

$$
\left|\Lambda^{+}-\frac{\gamma}{m \beta-1} \Lambda^{-}\right|<\Lambda^{+}
$$

From Lemma 1, it has

$$
\rho\left(\Lambda^{+}-\frac{\gamma}{m \beta-1} \Lambda^{-}\right)<\rho\left(\Lambda^{+}\right) \text {. }
$$

In the new model, $\sum_{k=-s}^{s} \lambda_{j, k}=1,0 \leq \lambda_{j, k} \leq 1$, and $0 \leq$ $P^{(j, k)} \leq 1$ for all $1 \leq j \leq s, 1 \leq|k| \leq s$. It suffices to prove that $\left\|\Lambda^{+}\right\|_{1}+\left\|\Lambda^{-}\right\| \leq 1$. Then, we obtain

$$
\rho\left(\Lambda^{+}-\frac{1}{m \beta-1} \Lambda^{-}\right)<\rho\left(\Lambda^{+}\right) \leq 1
$$

The new improved parsimonious multivariate Markov chain model is convergent.

\section{Estimation of the Parameters of the New Improved Parsimonious Multivariate Markov Chain Model}

In this section, we estimate the parameters of the new improved parsimonious multivariate Markov chain model in the new convergence condition with the new variability which has been proved in Theorem 2 . The transition probability matrices $P^{(j, k)}$ are estimated at first. If the data sequences are given and the state set is $\mathscr{M}=\{1,2, \ldots, m\}, f_{i_{j}, i_{k}}^{(j, k)}$ is the frequency from the $i_{k}$ state in the $k$ th sequence at time $t$ to the $i_{j}$ state in the $j$ th sequence at time $t+1$ with $i_{j}, i_{k} \in \mathscr{M}$, then the transition frequency matrix $F^{(j, k)}$ can be constructed as follows:

$$
F^{(j, k)}=\left(\begin{array}{cccc}
f_{1,1}^{(j, k)} & f_{1,2}^{(j, k)} & \cdots & f_{1, m}^{(j, k)} \\
f_{2,1}^{(j, k)} & f_{2,2}^{(j, k)} & \cdots & f_{2, m}^{(j, k)} \\
\vdots & \vdots & \vdots & \vdots \\
f_{m, 1}^{(j, k)} & f_{m, 2}^{(j, k)} & \cdots & f_{m, m}^{(j, k)}
\end{array}\right)_{m \times m} .
$$

Here, $P^{(j, k)}$ can be obtained by normalizing the frequency transition probability matrix as

$$
P^{(j, k)}=\left(\begin{array}{cccc}
p_{1,1}^{(j, k)} & p_{1,2}^{(j, k)} & \cdots & p_{1, m}^{(j, k)} \\
p_{2,1}^{(j, k)} & p_{2,2}^{(j, k)} & \cdots & p_{2, m}^{(j, k)} \\
\vdots & \vdots & \vdots & \vdots \\
p_{m, 1}^{(j, k)} & p_{m, 2}^{(j, k)} & \cdots & p_{m, m}^{(j, k)}
\end{array}\right)_{m \times m},
$$

where

$$
p_{i_{j}, i_{k}}^{(j, k)}= \begin{cases}\frac{f_{i_{j}, i_{k}}^{(j, k)}}{\sum_{i_{j}=1}^{m} f_{i_{j}, i_{k}}^{(j, k)}} & \text { if } \sum_{i_{j}=1}^{m} f_{i_{j}, i_{k}}^{(j, k)} \neq 0, \\ \frac{1}{m} & \text { otherwise. }\end{cases}
$$

Subsequently, the way of estimating the parameter $\lambda_{j, k}$ is introduced. $X_{t}$ is a joint state probability distribution of the new improved parsimonious multivariate Markov chain model at $t$ and can be represented as

$$
X_{t}=\left(\left(X_{t}^{(1)}\right)^{\mathrm{T}},\left(X_{t}^{(2)}\right)^{\mathrm{T}}, \ldots\left(X_{t}^{(s)}\right)^{\mathrm{T}}\right)^{\mathrm{T}} \in \mathbb{R}^{m s \times 1}
$$

which satisfies

$$
X_{t+1}=\Lambda^{+} X_{t}+\frac{1}{m \beta-1} \Lambda^{-}\left(\beta \mathbf{e}-X_{t}\right)
$$

where $\Lambda^{+}, \Lambda^{-}$have been denoted in Section 3 satisfying

$$
\sum_{j=-s}^{s} \lambda_{j, k}=1, \quad \lambda_{j, k} \geq 0, \text { for } \forall 1 \leq j \leq s, 1 \leq|k| \leq s
$$

and $\beta<1 / \mathrm{m}$. Based on the idea of the convergence condition in [5], the iteration matrix of the new improved parsimonious multivariate Markov chain model $M_{s}$ satisfies

$$
\left\|M_{s}\right\|_{\infty} \leq \max _{1 \leq k \leq s}\left\{m\left|\lambda_{k, k}-\frac{\lambda_{k,-k}}{(m \beta-1)}\right|+\sum_{k \neq i}\left|\lambda_{k, i}-\frac{\lambda_{k,-i}}{(m \beta-1)}\right|\right\} .
$$

By imposing an upper bound $\alpha<1$, the convergence condition of the new improved parsimonious multivariate Markov chain model is

$$
\begin{array}{r}
m\left|\lambda_{k, k}-\frac{\lambda_{k,-k}}{(m \beta-1)}\right|+\sum_{k \neq i}\left|\lambda_{k, i}-\frac{\lambda_{k,-i}}{(m \beta-1)}\right| \leq \alpha \\
\text { for } i=1,2, \ldots, s .
\end{array}
$$

Then the new improved parsimonious multivariate Markov chain model in this convergence condition can be represented as a set of $s$ linear programming problems

$$
\min _{\lambda_{j, k}} \sum_{j} \omega_{j}
$$


subject to

$$
\begin{aligned}
& b_{j, k}=\sum_{k=1}^{s}\left(\left(\lambda_{j, k}-\frac{\lambda_{j,-k}}{m-1}\right) \triangle_{j, k} X_{t}+\frac{1}{m-1} \lambda_{j, k} \triangle_{j, k} \mathbf{e}\right), \\
& \omega_{j} \geq\left[b_{j, k}-X^{(j)}\right], \\
& \omega_{j} \geq-\left[b_{j, k}-X^{(j)}\right], \\
& \sum_{k=-s}^{s} \lambda_{j, k}=1 \quad \text { for } \forall 1 \leq j \leq s, \\
& \lambda_{j, k} \geq 0 \quad \text { for } \forall 1 \leq j \leq s, 1 \leq|k| \leq s, \\
& A_{j} \Lambda_{j} \leq \alpha \mathbf{e} \quad \text { for } \forall 1 \leq j \leq s, \beta<\frac{1}{m},
\end{aligned}
$$

where

$$
\begin{aligned}
\triangle_{j, k} & = \begin{cases}P^{(j, j)}, & \text { if } j=k, \\
I, & \text { if } j \neq k,\end{cases} \\
\Lambda_{j} & =\left(\lambda_{j, 1}, \ldots, \lambda_{j, s}, \lambda_{j,-1}, \ldots, \lambda_{j,-s}\right)^{\mathrm{T}}, \\
A_{j} & =\left[A_{1 j}, A_{2 j}\right], \\
A_{1 j} & =\left(\begin{array}{cccccc}
1 & \cdots & m & \cdots & 1 & 1 \\
1 & \cdots & m & \cdots & 1 & -1 \\
1 & \cdots & m & \cdots & -1 & 1 \\
\vdots & \vdots & \vdots & \vdots & \vdots & \vdots \\
\vdots & \vdots & \vdots & \vdots & \vdots & \vdots \\
-1 & \cdots & -m & \cdots & -1 & 1 \\
-1 & \cdots & \underbrace{-m}_{j \text { th }} & \cdots & -1 & -1
\end{array}\right), \\
A_{2 j} & =-\frac{1}{m \beta-1} A_{1 j} .
\end{aligned}
$$

Here, $A_{1 j}$ covers all of the rows of each component taking one of the two possible values, 1 and -1 , particularly, in $j$ th column taking $m$ and $-m$. Then $A=\left[A_{j}\right]$ has $s \times 2^{s}$ rows. This convergence condition is viable only when the sequence group is not large.

To speed up the convergence of the new improved parsimonious multivariate Markov chain model and minimize the scale of the convergence condition, we apply a new convergence condition $B>(\gamma /(m \beta-1)) C$ with $\gamma \geq 1$ and $\beta<1 / m$ for the new model. Then, the form of the new improved parsimonious multivariate Markov chain model can be represented as

$$
\min _{\lambda_{j, k}}\left|f^{(j)}-X^{(j)}\right|_{j}
$$

subject to:

$$
\begin{aligned}
& f^{(j)}=\sum_{k=1}^{s} \lambda_{j, k} P^{(j, k)} X^{(k)}+\frac{1}{m \beta-1} \sum_{k=1}^{s} \lambda_{j, k} P^{(j, k)}\left(\beta \mathbf{e}-X^{(k)}\right), \\
& \sum_{k=-s}^{s} \lambda_{j, k}=1 \quad \text { for } \forall 1 \leq|k| \leq s, \\
& \lambda_{j, k} \geq 0 \quad \text { for } \forall 1 \leq j \leq s, 1 \leq|k| \leq s, \\
& \lambda_{j, k}>\frac{\gamma}{m \beta-1} \lambda_{j,-k} \quad \text { for } \forall 1 \leq j \leq s, 1 \leq|k| \leq s, \\
& \gamma \geq 1, \beta<\frac{1}{m},
\end{aligned}
$$

where $[\cdot]_{i}$ is the $i$ th entry of the vector. Certainly, the optimization problem can also be represented as a linear programming problem:

$$
\min _{\lambda_{j, k}} \sum_{j} \omega_{j}
$$

subject to

$$
\begin{aligned}
& f^{(j)}=\sum_{k=1}^{s} \lambda_{j, k} P^{(j, k)} X^{(k)}+\frac{1}{m \beta-1} \sum_{k=1}^{s} \lambda_{j, k} P^{(j, k)}\left(\beta \mathbf{e}-X^{(k)}\right), \\
& \omega_{j} \geq\left[f^{(j)}-X^{(j)}\right], \\
& \omega_{j} \geq-\left[f^{(j)}-X^{(j)}\right], \\
& \sum_{k=-s}^{s} \lambda_{j, k}=1 \quad \text { for } \forall 1 \leq|k| \leq s, \\
& \lambda_{j, k} \geq 0 \quad \text { for } \forall 1 \leq j \leq s, 1 \leq|k| \leq s, \\
& \lambda_{j, k}>\frac{\gamma}{m \beta-1} \lambda_{j,-k} \quad \text { for } \forall 1 \leq j \leq s, 1 \leq|k| \leq s, \\
& \quad \gamma \geq 1, \beta<\frac{1}{m} .
\end{aligned}
$$

\section{Numerical Experiments}

In this section, numerical experiments with three examples of different improved parsimonious multivariate Markov chain models with different convergence conditions are reported.

Noting that the new improved parsimonious multivariate Markov chain model with the original convergence condition [5] is "IPM1," (especially, the new improved parsimonious multivariate Markov chain model is an improved multivariate Markov chain model when we choose $\beta=1$ ), the new improved parsimonious multivariate Markov chain model with the new convergence condition of the new variability is "IPM2," the convergence factor of the original convergence condition is " $\alpha$ ", and the variabilities of the new convergence condition are " $\beta$ " and " $\gamma$." The stopping criterion can be found 
in Matlab order of linprog. We add a notation "*” at the back of data in Tables $1,2,3$, and 4 when the stopping criterion is satisied but the accuracy is not reached.

5.1. Example 1. There are three categorical data sequences [13]:

$$
\begin{aligned}
& S_{1}=\{1,2,2,1,3,3,2,3,1,3,3,2,2,3,2,1,2,1,2,2\}, \\
& S_{2}=\{2,2,1,1,2,1,2,1,2,2,2,2,3,2,2,1,2,1,2,2\}, \\
& S_{3}=\{3,1,1,2,2,1,2,3,1,1,3,3,3,2,3,2,3,3,1,2\} .
\end{aligned}
$$

IPM1 with $\alpha=0.7$ [5] can be represented as

$$
\begin{aligned}
x_{r+1}^{(1)}= & 0.3000 P^{(1,2)} x_{r}^{(2)}+0.4737 P^{(1,3)}\left(\mathbf{e}-x_{r}^{(3)}\right), \\
x_{r+1}^{(2)}= & 0.0088 P^{(2,1)} x_{r}^{(1)}+0.1912 P^{(2,1)}\left(\mathbf{e}-x_{r}^{(1)}\right) \\
& +0.8000 P^{(2,3)} x_{r}^{(3)}, \\
x_{r+1}^{(3)}= & 1.0000 P^{(3,3)} x_{r}^{(3)} .
\end{aligned}
$$

$$
x_{r+1}^{(3)}=0.0350 P^{(3,3)} x_{r}^{(3)}+0.9650 P^{(3,3)}\left(\mathbf{e}-x_{r}^{(3)}\right) .
$$

5.2. Example 2. Let the three categorical data sequences be

$$
\begin{aligned}
& S_{1}=\{1,4,3,3,1,1,3,4,4,3,2,3\}, \\
& S_{2}=\{2,3,4,4,4,2,3,3,1,4,3,2\}, \\
& S_{3}=\{3,3,1,1,3,1,4,3,2,4,3,2\} .
\end{aligned}
$$

Suppose that $\bar{X}_{t}=\left[\left(\bar{X}_{t}^{(1)}\right)^{\mathrm{T}},\left(\bar{X}_{t}^{(2)}\right)^{\mathrm{T}}, \ldots,\left(\bar{X}_{t}^{(s)}\right)^{\mathrm{T}}\right]^{\mathrm{T}}$ is the prediction probability at time $t$ and $X_{t}=$ $\left[\left(X_{t}^{(1)}\right)^{\mathrm{T}},\left(X_{t}^{(2)}\right)^{\mathrm{T}}, \ldots,\left(X_{t}^{(s)}\right)^{\mathrm{T}}\right]^{\mathrm{T}}$ is the fact value at time $t$ where $X_{t}^{(i)}=e_{\left(m_{t}\right)}=\{0, \ldots, 0,1,0, \ldots, 0\}^{\mathrm{T}} \in \mathbb{R}^{1 \times m}$, $i \in\{1, \ldots, s\}, m_{t}$ is the fact state at time $t$ in the $i$ th categorical

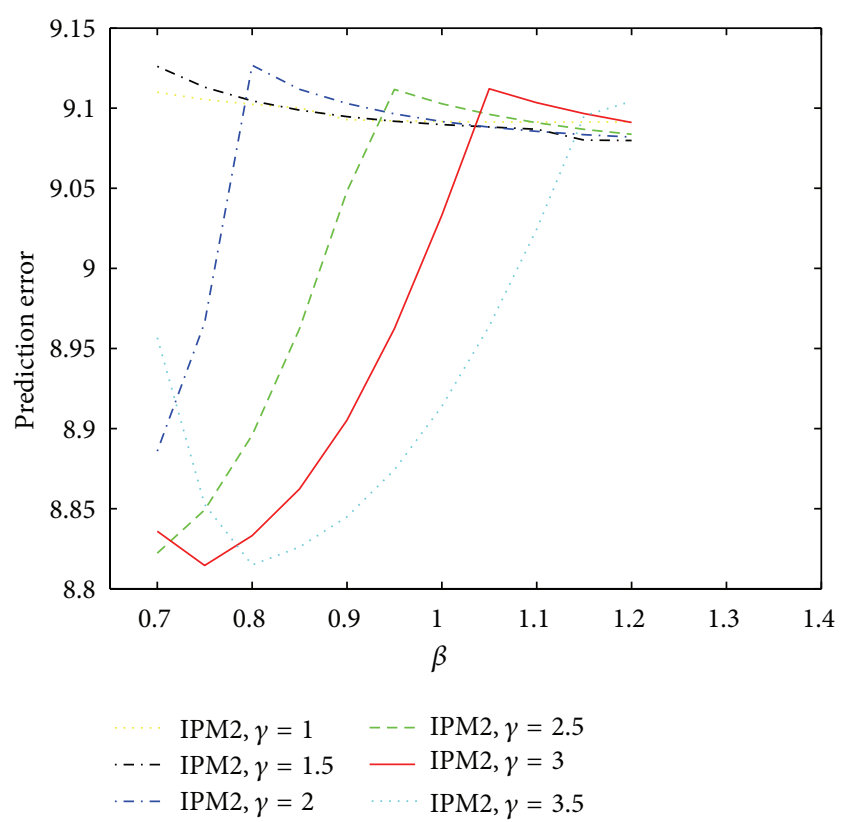

FIgURE 1: The prediction errors of the new improved parsimonious multivariate Markov chain model with our new convergence condition of the new variability in Example 2.

data sequence. " $\mathrm{nA}$ " is the number of the categorical data in one sequence and "pe" is the prediction error of the models which can be estimated by the equation:

$$
\mathrm{pe}=\sum_{t=4}^{\mathrm{nA}}\left\|\bar{X}_{t}-X_{t}\right\|_{2} \text {. }
$$

In Table 1, the prediction errors of the new improved parsimonious multivariate Markov chain model when $\beta=$ 1.2 is better than the prediction errors of other values of $\beta$. Table 1 illustrates the efficiency of the new improved parsimonious multivariate Markov chain model when $\beta=$ 1.2 in the original convergence condition [5].

In Table 2, numerical experiments on the prediction errors of the new improved parsimonious multivariate Markov chain model of the new convergence condition with the new variability " $\gamma$ " are reported. The best performance of the prediction errors of the new model with the new convergence condition of the new variability is 8.6790 when we choose $\beta=0.8$ and $\gamma=3$. In different cases of $\beta$, the best prediction errors of the new model with the new convergence condition of the new variability are in the diagonal line of the result matrix between $\gamma=2.5$ and $\gamma=5$.

For comparing the performances of the new improved parsimonious multivariate Markov chain model with the new convergence condition more clearly, we present Figure 1.

To compare the performances of the new improve parsimonious multivariate Markov chain model in different convergence conditions, we show Figure 2 with the data of Tables 1 and 2. Obviously, the results of the new model in the new convergence condition with the new variability are 
TABLE 1: Prediction errors of the new improved parsimonious multivariate Markov chain model with original convergence condition in Example 2.

\begin{tabular}{|c|c|c|c|c|c|c|}
\hline & $\beta=0.7$ & $\beta=0.8$ & $\beta=0.9$ & $\beta=1$ & $\beta=1.1$ & $\beta=1.2$ \\
\hline IPM1 when $\alpha=0.1$ & $31.1845^{*}$ & $31.0831^{*}$ & $29.8349^{*}$ & $29.8349^{*}$ & $31.0906^{*}$ & $31.0853^{*}$ \\
\hline IPM1 when $\alpha=0.2$ & $27.0472^{*}$ & $26.9434^{*}$ & $26.8745^{*}$ & $26.8745^{*}$ & $26.9486^{*}$ & $26.8462^{*}$ \\
\hline IPM1 when $\alpha=0.3$ & $22.8309^{*}$ & $22.7298^{*}$ & $22.7296^{*}$ & $22.7302^{*}$ & $22.7321^{*}$ & $22.7310^{*}$ \\
\hline IPM1 when $\alpha=0.4$ & $18.6215^{*}$ & $18.5266^{*}$ & $18.5256^{*}$ & $18.5256^{*}$ & $18.5282^{*}$ & $18.5211^{*}$ \\
\hline IPM1 when $\alpha=0.5$ & $14.3298^{*}$ & $14.2594^{*}$ & $14.2614^{*}$ & $14.2614^{*}$ & $14.2586^{*}$ & $14.2705^{*}$ \\
\hline IPM1 when $\alpha=0.6$ & $10.4531^{*}$ & $10.2961^{*}$ & $10.3231^{*}$ & $10.2962^{*}$ & $10.2795^{*}$ & $10.2971^{*}$ \\
\hline IPM1 when $\alpha=0.7$ & 9.9017 & 9.8994 & 9.8872 & 9.8879 & 9.8896 & 9.8552 \\
\hline IPM1 when $\alpha=0.8$ & 10.1173 & 10.1440 & 10.1255 & 10.1271 & 10.1283 & 10.0536 \\
\hline IPM1 when $\alpha=0.9$ & 10.3910 & 10.4131 & 10.3945 & 10.3886 & 10.3803 & 10.2726 \\
\hline IPM1 when $\alpha=1$ & 10.4059 & 10.4268 & 10.4059 & 10.4059 & 10.4059 & 10.2590 \\
\hline
\end{tabular}

TABLE 2: Prediction errors of the new improved parsimonious multivariate Markov chain model with the new convergence condition of the new variability in Example 2.

\begin{tabular}{|c|c|c|c|c|c|c|}
\hline & $\beta=0.7$ & $\beta=0.8$ & $\beta=0.9$ & $\beta=1$ & $\beta=1.1$ & $\beta=1.2$ \\
\hline IPM2 when $\gamma=1$ & 8.9588 & 9.1114 & 9.0964 & 9.0894 & 9.0860 & 9.0778 \\
\hline IPM2 when $\gamma=1.5$ & 8.8126 & 8.8788 & 9.0326 & 9.1028 & 9.0904 & 9.0832 \\
\hline IPM2 when $\gamma=2$ & 8.9746 & 8.8142 & 8.8544 & 8.9447 & 9.0892 & 9.0974 \\
\hline IPM2 when $\gamma=2.5$ & 8.6957 & 8.9355 & 8.8167 & 8.8449 & 8.9054 & 9.0014 \\
\hline IPM2 when $\gamma=3$ & 8.9006 & 8.6790 & 8.7050 & 8.8189 & 8.8410 & 8.8849 \\
\hline IPM2 when $\gamma=3.5$ & 9.4994 & 8.7730 & 8.6838 & 8.7168 & 8.8209 & 8.8393 \\
\hline IPM2 when $\gamma=4$ & $9.1024^{*}$ & 9.1909 & 8.7041 & 8.6911 & 8.7275 & 8.8228 \\
\hline IPM2 when $\gamma=4.5$ & $9.3544^{*}$ & $9.5649^{*}$ & 9.0116 & 8.6994 & 8.6993 & 8.7371 \\
\hline IPM2 when $\gamma=5$ & $12.3474^{*}$ & $9.0286^{*}$ & 9.3882 & 8.8994 & 8.7029 & 8.7076 \\
\hline IPM2 when $\gamma=5.5$ & $13.5788^{*}$ & $9.4195^{*}$ & $9.4562^{*}$ & 9.1952 & 8.8252 & 8.7046 \\
\hline
\end{tabular}

much better than those of the new model in the original convergence condition.

5.3. An Application to Sales Demand Predictions. In this part, the sales demand sequences are presented to show the effectiveness of the new improved parsimonious multivariate Markov chain model of the new convergence condition with the new variability. Since the requirement of the market fluctuates heavily, the production planning and the inventory control directly affect the estate cost. Thus, studying the interplay between the storage space requirement and the overall growing sales demand is a pressing issue for the company. Suppose that the products are classified into six possible states $(1,2,3,4,5,6)$, for example, $1=$ no sales volume, 2 = very low sales volume, 3 = low sales volume, $4=$ standard sales volume, 5 = fast sales volume, and 6 = very fast sales volume. The data of customer's sales demand of five important products can be found in [3].

By computing the proportion of the occurrence of each state in the sequences, the initial probability distributions of the five categorical data sequences are

$$
\begin{aligned}
& x_{0}^{(1)}=(0.0818,0.4052,0.0483,0.0335,0.0037,0.4275)^{\mathrm{T}}, \\
& x_{0}^{(2)}=(0.3680,0.1970,0.0335,0.0000,0.0037,0.3978)^{\mathrm{T}}, \\
& x_{0}^{(3)}=(0.1450,0.2045,0.0186,0.0000,0.0037,0.6283)^{\mathrm{T}},
\end{aligned}
$$

$$
\begin{aligned}
& x_{0}^{(4)}=(0.0000,0.3569,0.1338,0.1896,0.0632,0.2565)^{\mathrm{T}}, \\
& x_{0}^{(5)}=(0.0000,0.3569,0.1227,0.2268,0.0520,0.2416)^{\mathrm{T}} .
\end{aligned}
$$

The transition probability matrix $P^{(j, k)}$ can be obtained after normalizing the transition frequency matrix. By solving the linear programming problem corresponding to the new improved parsimonious multivariate Markov chain model with the new convergence condition where $\beta=0.7$ and $\gamma=4$, $\lambda_{i, j}$ is obtained and the model is presented as follows:

$$
\begin{aligned}
x_{r+1}^{(1)}= & 0.4359 P^{(1,1)} x_{r}^{(1)}+0.0193 P^{(1,2)}\left(\mathrm{e} * 0.7-x_{r}^{(2)}\right) \\
& +0.1210 P^{(1,3)}\left(\mathrm{e} * 0.7-x_{r}^{(3)}\right) \\
& +0.0359 P^{(1,5)}\left(\mathrm{e} * 0.7-x_{r}^{(5)}\right), \\
x_{r+1}^{(2)}= & 0.4950 P^{(2,1)} x_{r}^{(1)}+0.2416 P^{(2,3)} x_{r}^{(3)}+0.1189 P^{(2,4)} x_{r}^{(4)} \\
& +0.1445 P^{(2,5)}\left(\mathrm{e} * 0.7-x_{r}^{(5)}\right), \\
x_{r+1}^{(3)}= & 0.7748 P^{(3,1)} x_{r}^{(1)}+0.2252 P^{(3,2)} x_{r}^{(2)},
\end{aligned}
$$


TABLE 3: Prediction errors of the new improved parsimonious multivariate Markov chain model with the original convergence condition in sales demand prediction.

\begin{tabular}{lcccccc}
\hline & $\beta=0.7$ & $\beta=0.8$ & $\beta=0.9$ & $\beta=1$ & $\beta=1.1$ & $\beta=1.2$ \\
\hline IPM1 when $\alpha=0.1$ & $1624.2^{*}$ & $1628.5^{*}$ & $1631.7^{*}$ & $1634.1^{*}$ & $1636.1^{*}$ & $1637.7^{*}$ \\
IPM1 when $\alpha=0.2$ & $1354.0^{*}$ & $1355.8^{*}$ & $1359.3^{*}$ & $1361.1^{*}$ & $1363.8^{*}$ & $1365.1^{*}$ \\
IPM1 when $\alpha=0.3$ & $1083.7^{*}$ & $1086.4^{*}$ & $1088.4^{*}$ & $1089.9^{*}$ & $1091.1^{*}$ & $8^{*}$ \\
IPM1 when $\alpha=0.4$ & $809.2156^{*}$ & $811.0515^{*}$ & $812.4306^{*}$ & $813.5030^{*}$ & $815.0606^{*}$ \\
IPM1 when $\alpha=0.5$ & $543.7497^{*}$ & $545.0841^{*}$ & $546.0720^{*}$ & $546.8347^{*}$ & $547.4426^{*}$ & $547.9362^{*}$ \\
IPM1 when $\alpha=0.6$ & 366.9729 & 366.9684 & 366.9580 & 366.9577 & 366.9533 & 366.9589 \\
IPM1 when $\alpha=0.7$ & 367.3149 & 367.2101 & 367.1579 & 367.1367 & 367.1448 & 367.1498 \\
IPM1 when $\alpha=0.8$ & 367.5954 & 367.5916 & 367.5874 & 367.5769 & 367.5771 & 367.5766 \\
IPM1 when $\alpha=0.9$ & 368.2437 & 368.2422 & 368.2464 & 368.2530 & 368.2550 & 368.2639 \\
IPM1 when $\alpha=1$ & 369.1255 & 369.1145 & 369.1161 & 369.1119 & 369.1088 & 369.1062 \\
\hline
\end{tabular}

TABLE 4: Prediction errors of the new improved parsimonious multivariate Markov chain model with the new convergence condition of the new variability in sales demand prediction.

\begin{tabular}{|c|c|c|c|c|c|c|}
\hline & $\beta=0.7$ & $\beta=0.8$ & $\beta=0.9$ & $\beta=1$ & $\beta=1.1$ & $\beta=1.2$ \\
\hline IPM2 when $\gamma=1$ & 348.9387 & 348.8616 & 348.6692 & 348.4583 & 346.8533 & 347.9374 \\
\hline IPM2 when $\gamma=2$ & 341.3333 & 348.4608 & 348.5667 & 348.6407 & 348.6676 & 348.6457 \\
\hline IPM2 when $\gamma=3$ & 333.1122 & 334.4701 & 339.0412 & 343.5102 & 348.3476 & 348.3801 \\
\hline IPM2 when $\gamma=4$ & 334.2837 & 331.1873 & 333.7044 & 334.5513 & 337.5047 & 340.6662 \\
\hline IPM2 when $\gamma=5$ & $333.1028^{*}$ & 332.8355 & 330.7546 & 332.4308 & 333.8498 & 334.6122 \\
\hline IPM2 when $\gamma=6$ & $327.5181^{*}$ & $333.2228^{*}$ & 335.7616 & 331.0009 & 331.4727 & 332.7485 \\
\hline IPM2 when $\gamma=7$ & $481.5991^{*}$ & $327.9535^{*}$ & $333.2418^{*}$ & 335.7036 & 334.4605 & 331.1581 \\
\hline IPM2 when $\gamma=8$ & $550.2992^{*}$ & $457.7242^{*}$ & $329.7488^{*}$ & $333.3142^{*}$ & 335.7388 & 333.6250 \\
\hline IPM2 when $\gamma=9$ & $618.8261^{*}$ & $521.8871^{*}$ & $452.3191^{*}$ & $331.4777^{*}$ & $333.4008^{*}$ & 335.0657 \\
\hline IPM2 when $\gamma=10$ & $527.6297^{*}$ & $579.7398^{*}$ & $501.1963^{*}$ & $442.6577^{*}$ & $331.6313^{*}$ & $333.5236^{*}$ \\
\hline
\end{tabular}

$$
\begin{aligned}
x_{r+1}^{(4)}= & 0.3554 P^{(4,1)} x_{r}^{(1)}+0.1354 P^{(4,3)}\left(\mathbf{e} * 0.7-x_{r}^{(3)}\right) \\
& +0.0661 P^{(4,5)}\left(\mathbf{e} * 0.7-x_{r}^{(5)}\right), \\
x_{r+1}^{(5)}= & 0.0288 P^{(5,1)}\left(\mathbf{e} * 0.7-x_{r}^{(1)}\right)+0.1121 P^{(5,3)} x_{r}^{(3)} \\
& +0.1927 P^{(5,3)}\left(\mathbf{e} * 0.7-x_{r}^{(3)}\right) \\
& +0.0560 P^{(5,4)}\left(\mathbf{e} * 0.7-x_{r}^{(4)}\right) .
\end{aligned}
$$

Here, the prediction error equation are represented as

$$
\mathrm{pe}=\sum_{t=9}^{\mathrm{nA}}\left\|\bar{X}_{t}-X_{t}\right\|_{2} \text {. }
$$

In Table 3, we present the prediction errors of the new improved parsimonious multivariate Markov chain model with the original convergence condition [5]. From the data of Table 3, we get Figure 3. In Figure 3, it is obvious that the new improved parsimonious multivariate Markov chain model of the original convergence condition performs the best when we choose $\alpha=0.6$. The smaller $\alpha$ we choose, the better error prediction we get.

In Table 4, the best performance of the prediction errors of the new improved parsimonious multivariate model with

the new convergence condition in sales demand prediction is 331.1873 when $\beta=0.8$ and $\gamma=4$. In different cases of $\beta$, the best prediction errors are in the diagonal line of the result matrix between $\gamma=3$ and $\gamma=8$.

For comparing the performances of the new improved parsimonious multivariate model with the new convergence condition of the new variability more clearly, we present Figure 4 where the data are extracted from Table 4 . As the value of $\beta$ increases, the parameter $\gamma$ of the best performances of the prediction results increases. The best performances of the IPM2 are almost the same in different cases of $\beta$.

With the data of Tables 3 and 4 , we get Figure 5. It illustrates the benefits of the new parsimonious multivariate Markov chain model of the new convergence condition with the new variability in prediction accuracy.

\section{Conclusion}

In this paper, we present a new improved parsimonious multivariate Markov chain model and a new convergence condition with a new variability, which can enhance the the prediction accuracy of the models and save the computational estate. Numerical experiments with three examples illustrate that the new improved parsimonious multivariate Markov chain model of the new convergence condition with the new variability performs better than the improved parsimonious 


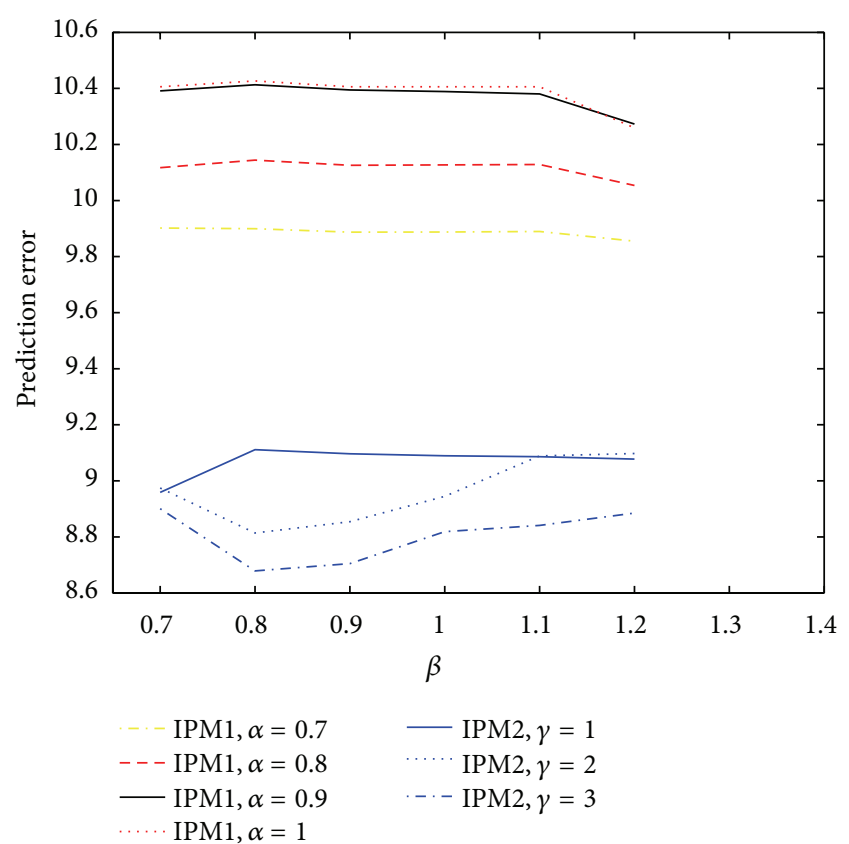

FIGURE 2: The prediction errors of the new improved parsimonious multivariate Markov chain model with the original convergence condition and the new convergence condition of the new variability in Example 2.

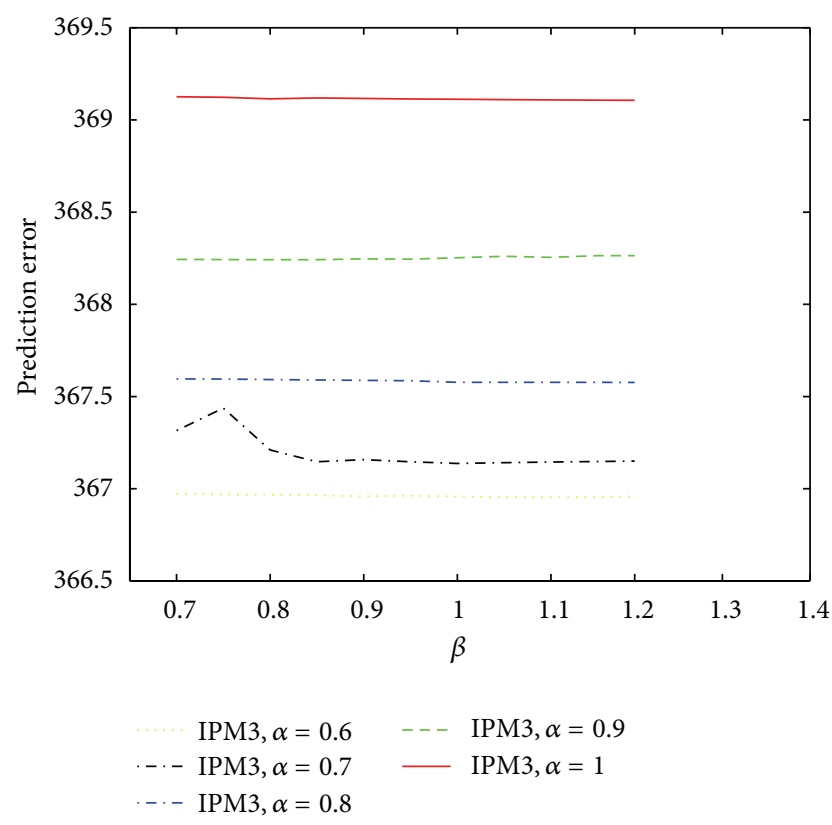

FIgURE 3: Prediction errors of the new improved parsimonious multivariate Markov chain model with the original convergence condition in sales demand prediction.

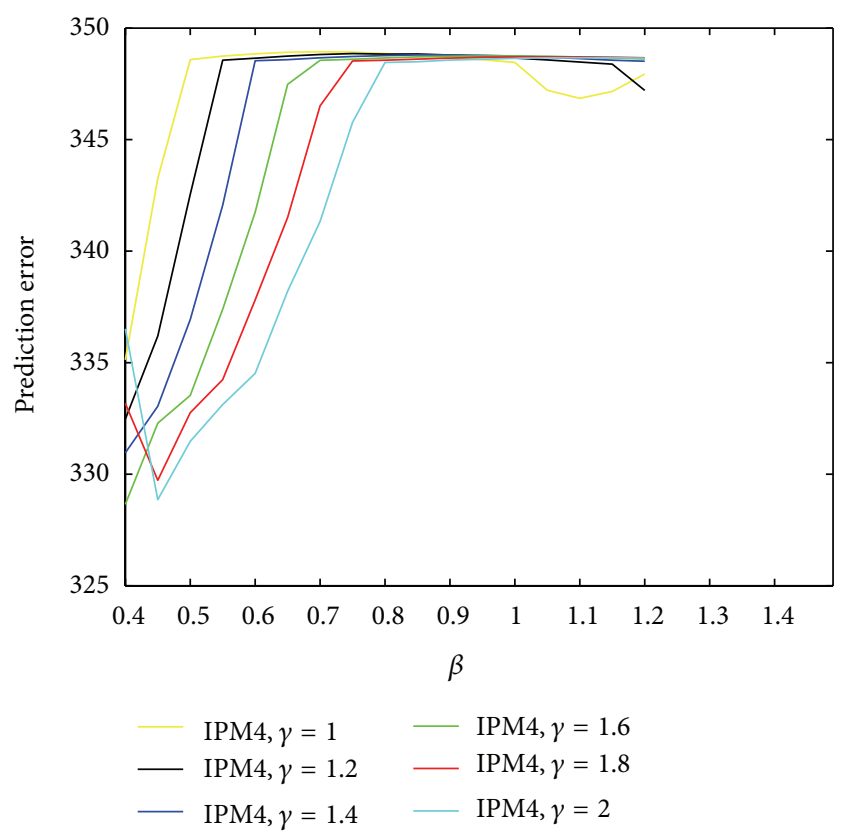

FIGURE 4: The prediction errors of the new improved parsimonious multivariate Markov chain model with the new convergence condition of the new variability in sales demand prediction.

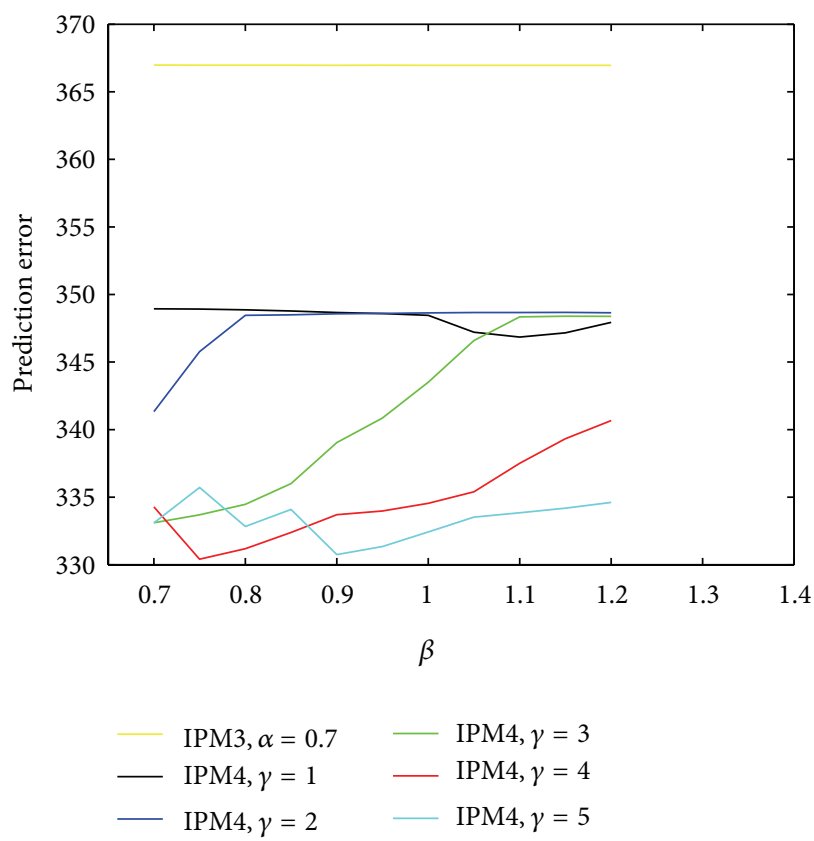

FIGURE 5: The prediction errors of the new improved parsimonious multivariate Markov chain model with the original convergence condition and the new convergence condition of the new variability in sales demand prediction. 
multivariate Markov chain model with the original convergence condition. Certainly, our new model can also be applied into credit risk and other research areas.

\section{Acknowledgments}

This research is supported by the Chinese Universities Specialized Research Fund for the Doctoral Program (20110185110020) and Sichuan Province Sci. \& Tech. Research Project (12ZC1802).

\section{References}

[1] W.-K. Ching and M. K. Ng, Markov Chains: Models, Algorithms and Applications, International Series on Operations Research and Management Science, Springer, New York, NY, USA, 2006.

[2] W.-K. Ching, E. S. Fung, and M. K. Ng, "A multivariate Markov chain model for categorical data sequences and its applications in demand predictions," IMA Journal of Management Mathematics, vol. 13, no. 3, pp. 187-199, 2002.

[3] W. K. Ching, E. S. Fung, and M. K. Ng, "Higher-order Markov chain models for categorical data sequences," Naval Research Logistics, vol. 51, no. 4, pp. 557-574, 2004.

[4] W. K. Ching, M. M. Ng, E. S. Fung, and T. Akutsu, "On construction of stochastic genetic networks based on gene expression sequences," International Journal of Neural Systems, vol. 15, no. 4, pp. 297-310, 2005.

[5] W. Ching, T. Siu, and L. Li, "An improved parsimonious multivariate Markov chain model for credit risk," Journal of Credit Risk, vol. 5, pp. 1-25, 2009.

[6] D. W. C. Miao and B. M. Hambly, "Recursive formulas for the default probability distribution of a heterogeneous group of defauleable entities," 2012.

[7] W.-K. Ching, M. K. Ng, and E. S. Fung, "Higher-order multivariate Markov chains and their applications," Linear Algebra and Its Applications, vol. 428, no. 2-3, pp. 492-507, 2008.

[8] C. Wang, T. Z. Huang, and C. Wen, "A simplified higher-order multivariate Markov chains model," submitted.

[9] C. Wang and T. Z. Huang, "Improved multivariate Markov chain model with the new convergence condition," submitted.

[10] T.-K. Siu, W.-K. Ching, E. S. Fung, and M. K. Ng, "On a multivariate Markov chain model for credit risk measurement," Quantitative Finance, vol. 5, no. 6, pp. 543-556, 2005.

[11] R. A. Horn and C. R. Johnson, Matrix Analysis, Cambridge University Press, Cambridge, UK, 1985.

[12] Z.-Y. You and C.-L. Wang, "A concept of nonlinear block diagonal dominance," Journal of Computational and Applied Mathematics, vol. 83, no. 1, pp. 1-10, 1997.

[13] C. Wang, T. Z. Huang, and W. K. Ching, "On simplified parsimonious models for higher-order multivariate Markov chains," submitted. 


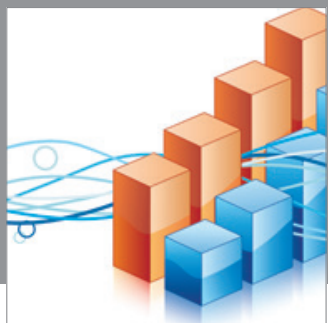

Advances in

Operations Research

mansans

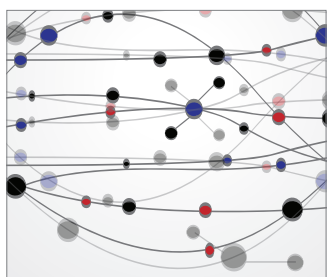

The Scientific World Journal
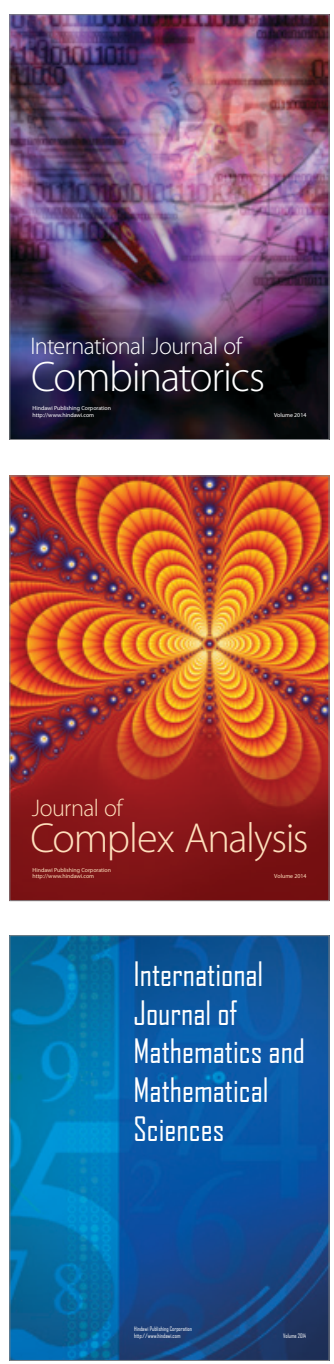


Submit your manuscripts at http://www.hindawi.com
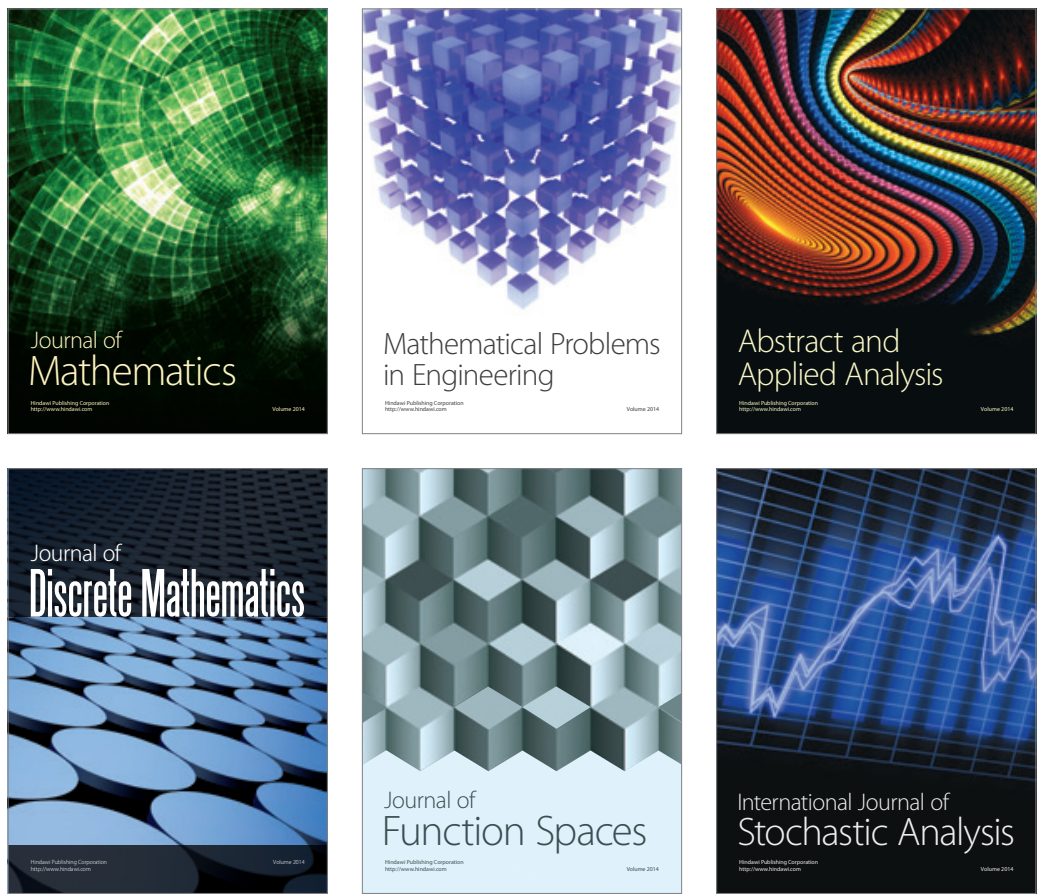

Journal of

Function Spaces

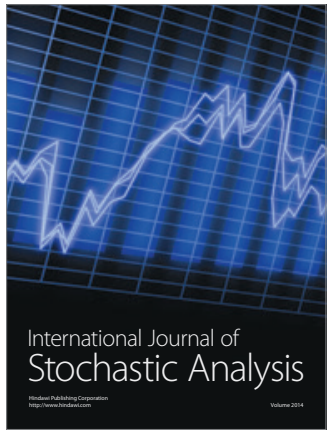

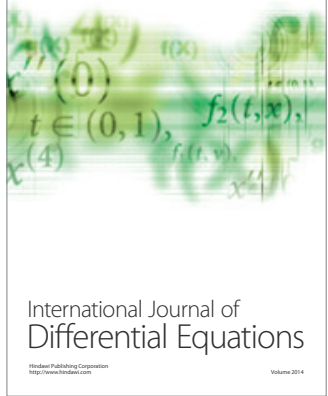
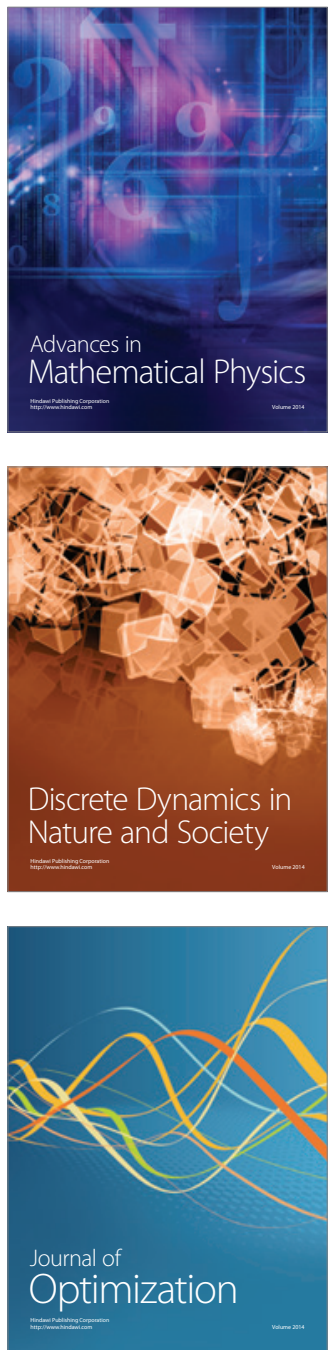